\title{
QCD Phase Diagram in Strong Coupling Lattice QCD with Polyakov Loops
}

\section{Kohtaroh Miura*}

INFN Laboratori Nazionali di Frascati, I-00044, Frascati (RM), Italy

E-mail: Kohtaroh.Miuradlnf.infn.it

\section{Takashi Z. Nakano}

Department of Physics, Faculty of Science, Kyoto University, Kyoto 606-8502, Japan Yukawa Institute for Theoretical Physics, Kyoto University, Kyoto 606-8502, Japan

\section{Akira Ohnishi}

Yukawa Institute for Theoretical Physics, Kyoto University, Kyoto 606-8502, Japan

\section{Noboru Kawamoto}

Department of Physics, Faculty of Science, Hokkaido University, Sapporo 060-0810, Hokkaido, Japan

\begin{abstract}
The chiral and deconfinement dynamics in QCD phase diagram are investigated by using the strong-coupling expansion of the lattice QCD with one species of staggered fermion and the Polyakov loop effective action at finite temperature $(T)$ and quark chemical potential $(\mu)$. Effective potential $\mathscr{F}_{\text {eff }}$ involves both the chiral $\left(U_{\chi}(1)\right)$ and the deconfinement $\left(Z_{N_{c}}\right)$ dynamics with finite coupling effects in the mean-field approximation. The Polyakov loop increasing rate $\left(d \ell_{p} / d T\right)$ and the susceptibility are found to have two peaks as a function of $T$ for small quark masses. One of them is the chiral-induced peak associated with the rapid decrease of the chiral condensate. The other peak is almost independent of the quark mass or chemical potential, and it is interpreted as the $Z_{N_{c}}$-induced peak.
\end{abstract}

The XXIX International Symposium on Lattice Field Theory - Lattice 2011

July 10-16, 2011

Squaw Valley, Lake Tahoe, California

\footnotetext{
* Speaker.
} 


\section{Introduction}

QCD phase diagram at finite temperature $(T)$ and/or quark chemical potential $(\mu)$ has attracted much attention in the high energy and nuclear physics. Key ingredients characterizing the QCD phase diagram are two approximate symmetries - chiral and $Z_{N_{c}}$. They are independent symmetries to each other, while entanglements of them in dynamics is a non-trivial issue, and essential to understand the QCD phase diagram.

The lattice QCD Monte-Carlo (LQCD-MC) simulations at high $T$ and vanishing (or small) $\mu$ indicate that peak positions of chiral and Polyakov loop susceptibilities $\left(\chi_{\sigma, \ell_{p}}\right)$ are close to each other. The small separation of them could be explained as a consequence of the broad analytic behavior of the crossovers [1]. These observations give us a solid numerical base to consider the relation between the chiral and $Z_{N_{c}}$.

We expect that finite $\mu$ affects these two symmetries in different way, and must be helpful to understand the chiral and $Z_{N_{c}}$ relations. For this purpose, strong coupling expansion in the lattice QCD provides a useful framework with less contaminations from the notorious sign problem, and without any additional parameters than those in QCD. Many theoretical knowledge have been accumulated so far [2- 4], and the QCD phase diagram have been well investigated in the strongcoupling limit [5-7]. The coupling between the chiral condensate $\sigma$ and the Polyakov-loop $\ell_{p}$ was extracted in the strong-coupling limit by Ilgenfritz-Kripfganz and by Gocksch-Ogilvie (IKGO model) [8], and led to the invention of the Nambu-Jona-Lasino model with Polyakov-loops (PNJL model) [9]. Thus the basic mechanism for chiral and $Z_{N_{c}}$ entanglements in whole $T-\mu$ plane has been captured in the strong coupling limit.

Recently, the essential mechanism for the phase diagram evolution resulting from the increasing finite lattice couping $\left(\beta=2 N_{c} / g^{2}\right)$ has been elaborated [10, 11]. This proceedings aims at extending the finite coupling knowledge to include $Z_{N_{c}}$ deconfinement dynamics as well as the chiral, then it inevitably leads to an extended (finite $\beta$ ) version of the aforementioned IK-GO model [8]. By using this "Polyakov loop extended strong coupling lattice QCD" (P-SC-LQCD), we investigate the Polyakov loop increasing rate $\left(d \ell_{p} / d T\right)$ and the susceptibility, and elucidate the chiral and $Z_{N_{c}}$ deconfinement relations.

\section{Effective potential}

We briefly overview the derivation of the effective potential in the strong coupling expansion [10-12]. We start from the lattice QCD partition function with one species of staggered fermion $(\chi)$ with a current quark mass $\left(m_{0}\right)$ in the lattice unit $a=1$,

$$
\mathscr{Z}=\int \mathscr{D}\left[\chi, \bar{\chi}, U_{v}\right] \exp \left[\frac{-1}{2} \sum_{v, x}\left[\eta_{v, x} \bar{\chi}_{x} U_{v, x} \chi_{x+\hat{v}}-\eta_{v, x}^{-1}(\text { h.c. })\right]-m_{0} \sum_{x} \bar{\chi}_{x} \chi_{x}+\beta \sum_{P} \frac{U_{P}+U_{P}^{\dagger}}{2 N_{c}}\right],
$$

where $U_{v, x} \in S U\left(N_{c}\right)$ and $U_{P=\mu v, x}=\operatorname{tr}_{c}\left[U_{\mu, x} U_{v, x+\hat{\mu}} U_{\mu, x+\hat{v}}^{\dagger} U_{v, x}^{\dagger}\right]$ represent the link-variable and plaquette, and the staggered sign factor $\eta_{v, x}=\exp \left(\mu \delta_{v 0}\right)(-1)^{x_{0}+\cdots+x_{v-1}}$ contains the the lattice chemical potential $\mu$. We concentrate on the color $\mathrm{SU}\left(N_{c}=3\right)$ in the $3+1$ dimension $(d=3)$ in the later discussion. 
We perform following procedures to obtain the effective potential. The detailed derivations are found in our previous papers [10-12]. First, we perform the Taylor expansion in terms of $\beta$ in Eq. 2.10, and integrate out spatial link variables. For terms including staggered quarks, we take account of the leading, next-to-leading (NLO) and next-to-next-to-leading (NNLO) terms. For pure gluonic contributions, we consider the leading order Polyakov-loop effects. Second, in the staggered quark terms, we extract the leading order in the $1 / d$ expansion [2], which corresponds to the minimum quark number diagrams in each order of strong coupling expansions. Third, we bosonize the obtained effective actions by introducing several auxiliary fields, which are summarized in Table1, Forth, we integrate out the staggered quark fields $(\chi, \bar{\chi})$. Here the resultant quark determinant is evaluated by using the Matsubara method, then temporal link variables reduces into Polyakov loops. And final, we evaluate the integral over the temporal link variables by replacing the Polyakov loops with their constant mean fields. Then we get the following effective potential,

$$
\begin{aligned}
& \mathscr{F}_{\text {eff }}(\Phi ; T, \mu) \equiv-\left(T \log \mathscr{Z}_{\mathrm{LQCD}}\right) / N_{s}^{d}=\mathscr{F}_{\mathrm{eff}}^{\chi}+\mathscr{F}_{\mathrm{eff}}^{\mathrm{Pol}}+\mathscr{O}\left(1 / g^{6}, 1 / \sqrt{d}\right), \\
& \mathscr{F}_{\mathrm{eff}}^{\chi}=\frac{1}{2} b_{\sigma}^{\prime} \sigma^{2}+\frac{1}{2} \beta_{\tau}^{\prime} \bar{\psi}_{\tau} \psi_{\tau}+\frac{1}{2} \beta_{s}^{\prime} \bar{\psi}_{s} \psi_{s}+\beta_{\tau \tau} \bar{\psi}_{\tau \tau} \psi_{\tau \tau}+\beta_{s s} \bar{\psi}_{s s} \psi_{s s}+\frac{1}{2} \beta_{\tau s} \bar{\psi}_{\tau s} \psi_{\tau s}, \\
& -N_{c} E_{q}-N_{c} \log \sqrt{Z_{+} Z_{-}}-T\left(\log \mathscr{R}_{q}\left(E_{q}-\tilde{\mu}, \ell, \bar{\ell}\right)+\log \mathscr{R}_{q}\left(E_{q}+\tilde{\mu}, \bar{\ell}, \ell\right)\right), \\
& \mathscr{F}_{\text {eff }}^{\mathrm{Pol}}=-2 T d N_{c}^{2}\left(\frac{1}{g^{2} N_{c}}\right)^{1 / T} \bar{\ell}_{p} \ell_{p}-T \log \left[1-6 \bar{l}_{p, \mathbf{x}} l_{p, \mathbf{x}}-3\left(\bar{l}_{p, \mathbf{x}} l_{p, \mathbf{x}}\right)^{2}+4\left(l_{p, \mathbf{x}}^{N_{c}}+\bar{l}_{p, \mathbf{x}}^{N_{c}}\right)\right] \text {, }
\end{aligned}
$$

where $E\left(\tilde{m}_{q}(\sigma)\right)=\sinh ^{-1}\left[\tilde{m}_{q}(\sigma)\right]$ corresponds to the quark excitation energy, and we have defined

$$
\begin{aligned}
& \Phi=\left(\sigma, \bar{\psi}_{s s}, \psi_{s s}, \bar{\psi}_{\tau \tau}, \psi_{\tau \tau}, \bar{\psi}_{\tau s}, \psi_{\tau s}, \bar{\psi}_{\tau}, \psi_{\tau}, \bar{\psi}_{s}, \psi_{s}, \bar{\ell}, \ell\right), \\
& \mathscr{R}_{q}(x, y, \bar{y})=1+N_{c}\left(y e^{-x / T}+\bar{y} e^{-2 x / T}\right)+e^{-3 x / T} \\
& \left(\tilde{\mu}, \tilde{m}_{q}\right)=\left(\mu-\log \sqrt{Z_{+} / Z_{-}},\left(b_{\sigma}^{\prime} \sigma+m_{0}-\beta_{\tau \tau}\left(\bar{\psi}_{\tau \tau}+\psi_{\tau \tau}\right)\right) / \sqrt{Z_{+} Z_{-}}\right), \\
& \left(Z_{+}, Z_{-}\right)=\left(1+\beta_{\tau}^{\prime} \bar{\psi}_{\tau}+4 \beta_{\tau \tau} \sqrt{Z_{+} Z_{-}} \tilde{m}_{q} \bar{\psi}_{\tau \tau}, 1+\beta_{\tau}^{\prime} \psi_{\tau}+4 \beta_{\tau \tau} \sqrt{Z_{+} Z_{-}} \tilde{m}_{q} \psi_{\tau \tau}\right) .
\end{aligned}
$$

The coefficients of the auxiliary fields $\Phi$ in Eq. 2.3. are defined as

$$
\begin{aligned}
& b_{\sigma}^{\prime}=b_{\sigma}+2\left[\beta_{s s} \psi_{s s}+\beta_{\tau s} \bar{\psi}_{\tau s}+\beta_{s}^{\prime}\left(\psi_{s}+\bar{\psi}_{s}\right)\right] \\
& \left(\beta_{\tau}^{\prime}, \beta_{s}^{\prime}\right)=\left(\beta_{\tau}+\beta_{\tau s} \psi_{\tau s}, \beta_{s}+2 \beta_{s s} \bar{\psi}_{s s}\right), \\
& \left(b_{\sigma}, \beta_{\tau}, \beta_{s}, \beta_{\tau \tau}, \beta_{s s}, \beta_{\tau s}\right) \\
& =\left(\frac{d}{2 N_{c}}, \frac{d}{N_{c}^{2} g^{2}}\left(1+\frac{1}{2 g^{2}}\right), \frac{d(d-1)}{8 N_{c}^{4} g^{2}}\left(1+\frac{1}{2 g^{2}}\right), \frac{d}{2 N_{c}^{3} g^{4}}, \frac{d(d-1)(d-2)}{16 N_{c}^{7} g^{4}}, \frac{d(d-1)}{2 N_{c}^{5} g^{4}}\right) .
\end{aligned}
$$

The equilibrium is determined by the stationary condition, $\partial \mathscr{F}_{\text {eff }} / \partial \Phi=0 . \mathscr{F}_{\text {eff }}^{\chi}$ is responsible for the chiral-dynamics, and $\mathscr{F}$ eff Pol originates from the plaquette action and governs the $Z_{N_{c}}$ dynamics. The chiral and $Z_{N_{c}}$ dynamics communicate to each other through the quark determinant effects $\mathscr{R}_{q}$ in Eq. 2.3). This entanglement effect between the chiral and $Z_{N_{c}}$ has been pointed out in the strong-coupling limit [8], and incorporated into the PNJL model [9]. In the current formulation, the entanglements are modified by the finite coupling effects via $\left(\tilde{m}_{q}(\beta), \tilde{\mu}(\beta)\right)$.

The $\bar{\ell}_{p} \ell_{p}$ term in Eq. (2.4) gives large finite $T$ effects to $Z_{N_{c}}$ dynamics at finite $\beta$, and vanishes in the strong-coupling limit. In the previous work in the strong coupling limit, this quadratic term 
Table 1: The auxiliary fields (entries of first line) and corresponding mesonic composite fields (entries of second line) in the NNLO effective potential. Here we define the mesonic composite fields as $\left(M, V_{x}^{+}, V_{x}^{-}\right)$ $=\left(\bar{\chi} \chi, \bar{\chi}_{x} e^{\mu} U_{0, x} \chi_{x+\hat{0}}, \bar{\chi}_{x+\hat{0}} e^{-\mu} U_{0, x}^{\dagger} \chi_{x}\right),\left(W_{\mathbf{x}}^{+}, W_{\mathbf{x}}^{-}\right)=\left(\bar{\chi}_{x} e^{2 \mu} U_{0, x} U_{0, x+\hat{0}} \chi_{x+2 \hat{0}}, \bar{\chi}_{x+2 \hat{0}} e^{-2 \mu} U_{0, x+\hat{0}}^{\dagger} U_{0, x}^{\dagger} \chi_{x}\right)$, and $L_{\mathbf{x}}=N_{c}^{-1} \prod_{\tau} U_{\tau, \mathbf{x}}$.

\begin{tabular}{c|c|c|c|c|c|c}
\hline \hline$\sigma$ & $\left(\bar{\psi}_{s s}, \psi_{s s}\right)$ & $\left(\bar{\psi}_{\tau \tau}, \psi_{\tau \tau}\right)$ & $\left(\bar{\psi}_{\tau s}, \psi_{\tau s}\right)$ & $\left(\bar{\psi}_{\tau}, \psi_{\tau}\right)$ & $\left(\bar{\psi}_{s}, \psi_{s}\right)$ & $(\bar{\ell}, \ell)$ \\
\hline$-M$ & $\left(W^{+}, W^{-}\right)$ & $(M M, M M M M)$ & $\left(-V^{+} V^{-}, 2 M M\right)$ & $\left(-V^{+}, V^{-}\right)$ & $(M M, M M)$ & $(\bar{L}, L)$ \\
\hline \hline
\end{tabular}

is fixed to a constant to be consistent with the empirical value of the string tension [8]. The finite coupling property of the current formulation allows us to investigate the $\beta$ evolution of the $\bar{\ell}_{p} \ell_{p}$ term, i.e. finite $T$ effects of $Z_{N_{c}}$ dynamics, without introducing additional parameters.

\section{Results}

First, we show results for the NLO case by ignoring $1 / g^{4}$ effects in Eq. (2.10) [13]. As shown in our recent work [12], the critical temperature at zero chemical potential becomes closer to the LQCD-MC results in the coupling region $\beta \lesssim 4$. Therefore, we focus our attention to $\beta=4$. Then we discuss the stability of main conclusions for variations of $\beta$.

In the left panel of Fig. 1 the phase diagram at $\left(\beta, m_{0}\right)=(4,0)$ is shown in the lattice unit. We find the first- (solid red) and second-order (dashed red) chiral transition lines separated by the (tri-)critical point $(\mathrm{CP})$ at $\left(\mu_{\mathrm{CP}}, T_{\mathrm{CP}}\right)=(0.58,0.19)$. The result is qualitatively consistent with the previous SC-LQCD with NLO effects [10].

We investigate a Polyakov loop increasing rate $d \ell_{p} / d T$ along with the dashed line in the left panel of Fig.1. We find the double-peak structure "P" and "Q" as shown in the right panel of Fig.1. The peak "P" locates at the chiral phase transition, and is clearly interpreted as the chiral-induced peak. The strong correlation of the Polyakov loop and the chiral condensate emerges through their coupling in $\mathscr{R}_{q}$ in Eq. (2.3), and the Polyakov loop shows a rapid variation at any point on the chiral transition boundary except $T=0$. The similar peak to "Q" is observed in the whole range of $\mu$. The strength of this peak becomes weaker for smaller $\mu$, which is expressed by the dotted line. A double-peak structure has been reported in the model studies based on PNJL model [14].

The peak "Q" can be understood as a signal of the $Z_{N_{c}}$-induced crossover: $Z_{N_{c}}$ is the symmetry in the pure gluonic sector, and it becomes exact in the heavy quark mass limit. We also expect weak $\mu$ dependence of the $Z_{N_{c}}$ deconfinement transition, since $\mathscr{F}_{\text {eff }}^{\mathrm{Pol}}$ does not directly depend on quark chemical potential $\mu$. For larger masses, $m_{0}>0.05$, the two peaks merges to a single peak. This single peak grows with increasing $m_{0}$, and its temperature is nearly $m_{0}$ independent, $T \sim 0.52$, which is close to $T_{\mathrm{Q}}$ at smaller quark masses. The $Z_{N_{c}}$-induced nature of "Q" and the merged single peak are confirmed by the weak dependence of $T_{\mathrm{Q}}$ on $\mu$ and $m_{0}$. It is interesting to find that the $Z_{N_{c}}$ nature survives in the chiral limit or small mass region, and can be observed as a peak in $d \ell_{p} / d T$.

Next, we discuss the $\beta$ dependence of the two peak structure of $d \ell_{p} / d T$ at finite $\mu$. In the left panel of Fig. 2. we show $d \ell_{p} / d T$ for several $\beta$ at $\left(m_{0}, \mu\right)=(0.01,0.5)$. As indicated from this figure, the two peaks are found at least in the range $2 \leq \beta \leq 6$. For each $\beta$, we define the 

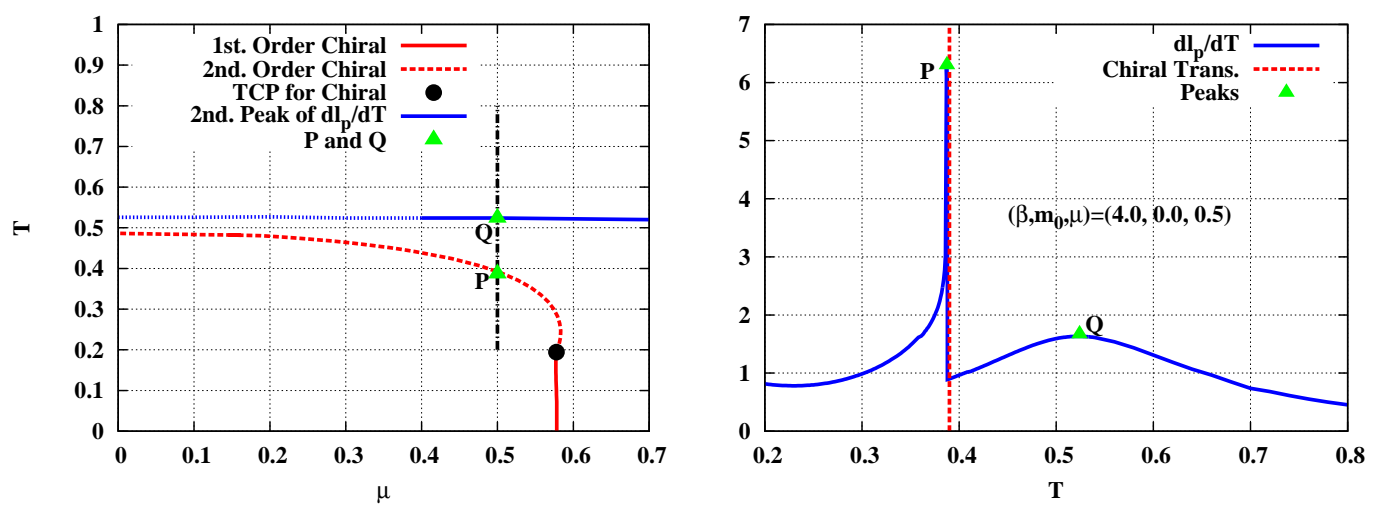

Figure 1: The NLO results in the lattice unit. Left: The phase boundary for the chiral transition with the second peak of $d \ell_{p} / d T$ at $\beta=4$ in the chiral limit. Right: $T$ dependence of $d \ell_{p} / d T$ at $\mu=0.5$ for $\beta=4$ in the chiral limit. The "P" and "Q" in the left figure correspond those of the right.

pseudo-critical temperatures for the chiral-induced $\left(T_{c, \mu=0.5}^{(\chi)}(\beta)\right)$ and $Z_{N_{c}}$-induced $\left(T_{c, \mu=0.5}^{(d)}(\beta)\right)$ deconfinement crossovers as the first and second peaks of $d \ell_{p} / d T$, respectively. Both of them are decreasing functions of $\beta$. For $T_{c, \mu=0.5}^{(\chi)}(\beta)$, the current results are close to the pseudo-critical temperatures for the chiral phase transition in our previous works [10, 11]. This again indicates the chiral induced nature of the first peak "P".

As shown in the right panel of Fig. 2, the double-peak structure is find in the Polyakov loop susceptibility in the region $3 \leq \beta \leq 6$. The peak positions are very close to those in $d \ell_{p} / d T$ for each $\beta$. The interesting point is that the second $\left(Z_{N_{c}}\right.$-induced) peak has much stronger signal than the first (chiral-induced). In summary for the NLO results, the two crossovers (chiral- and $Z_{N_{c}}$-induced) emerge in the large $\mu$ and small $m_{0}$ region as a consequence of chiral and $Z_{N_{c}}$ entanglements.
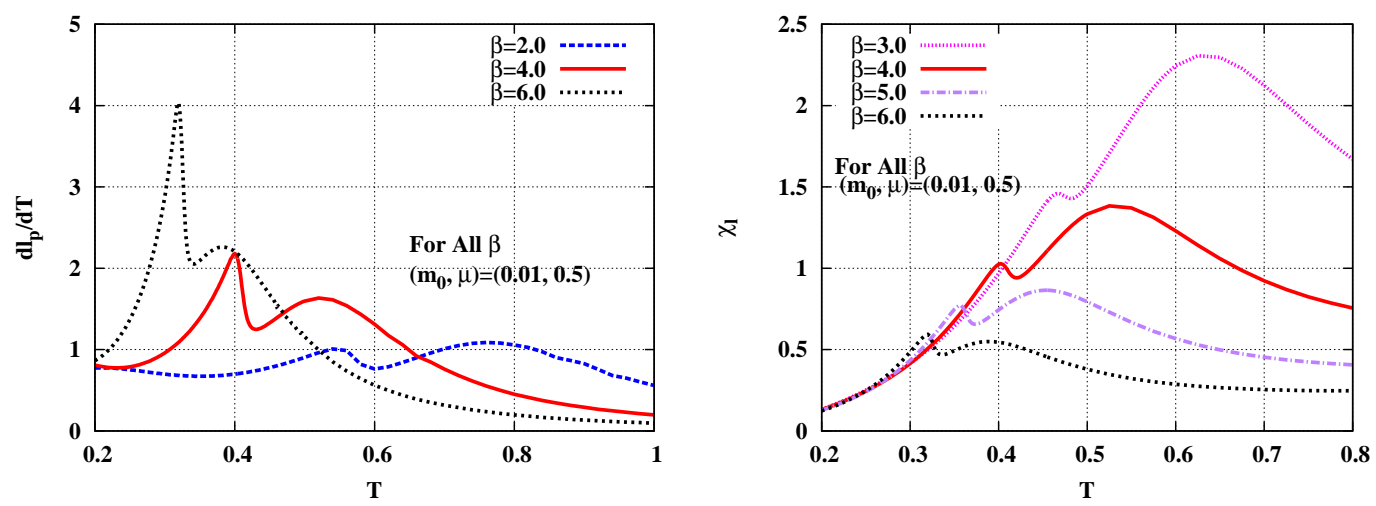

Figure 2: The NLO results in the lattice unit. The $T$ dependence of $d \ell_{p} / d T$ (left) and the Polyakov loop susceptibility (right) for several $\beta$ at $\left(m_{0}, \mu\right)=(0.01,0.5)$.

We extend our analyses to the NNLO cases. The chiral phase transition line is shown in the left panel of Fig. 3. We find that the critical temperature is strongly suppressed by the Polyakov- 
loop effects in whole region of the phase boundary. We shall consider the dimensionless ratio $R=\mu_{c, \mathrm{~T}=0} / T_{c, \mu=0}$. One of the problem in the strong coupling expansion has been that $R$ becomes much smaller than the empirical range $\sim 1.5-2.5$. As shown in the previous NLO work [10], $R$ is strongly enhanced by the finite coupling effects. Now we find further enhancement of $R$ due to the Polyakov loop effects: $R$ becomes larger than 1 in $\beta \sim 4$ and grows up to $\sim 1.6$ at $\beta=6$. These findings imply that the Polyakov loop effects play an important role for going closer to the continuum limit.

Finally, we investigate a fate of double-peak structures of $d \ell_{p} / d T$ in NNLO case. The right panel of Fig. 3 3 shows that the chiral- and $Z_{N_{c}}$-induced peaks still survive at $\beta=4.0$. The separation of two peaks becomes larger than the NLO case. The double-peak structure would be controversial to the recent works based on PNJL model combined with the statistical model [15] or PolyakovQuark Meson model with the functional renormalization group evolution [16].
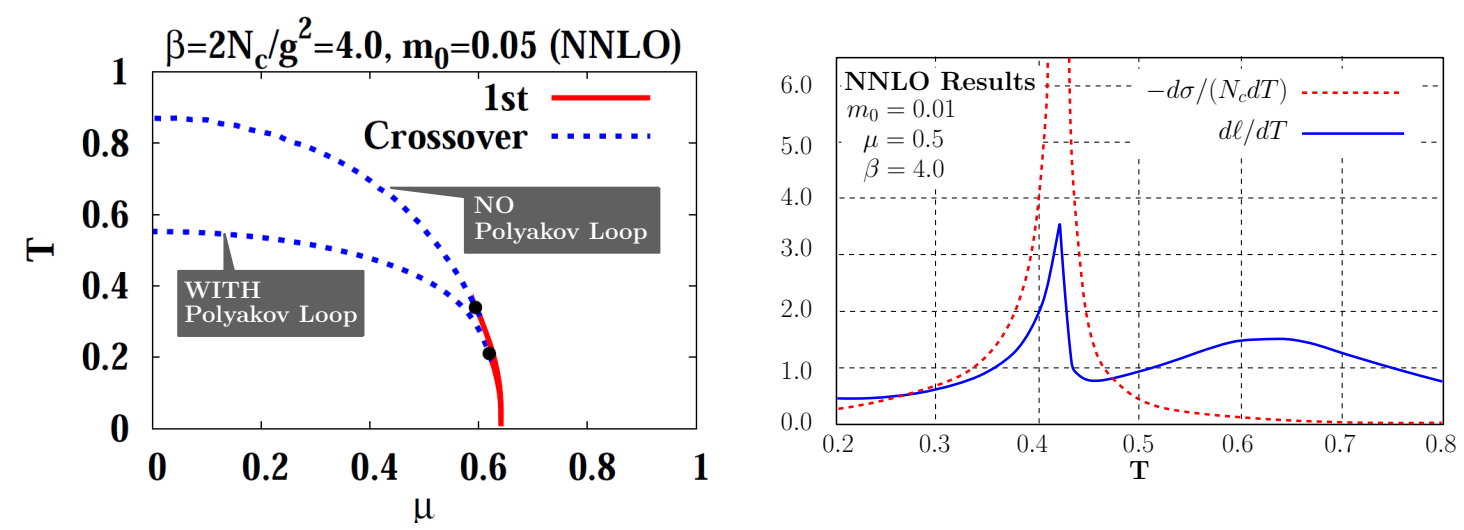

Figure 3: The NNLO results in the lattice unit. Left: The phase diagram at $\left(\beta, m_{0}\right)=(4.0,0.05)$. The crossover line (dashed blue line) is shifted to the lower $T$ direction due to the Polyakov loop effects. Right: The Polyakov loop increasing rate $d \ell_{p} / d T$ at $\left(\beta, m_{0}, \mu\right)=(4.0,0.01,0.5)$.

\section{Summary}

We have investigated the chiral and deconfinement crossovers at finite temperature $T$ and quark chemical potential $\mu$ based on the strong-coupling expansion in the lattice QCD with one species of staggered fermion. We have taken account of the leading, NLO and NNLO effects in the strong-coupling expansion for fermionic sector, and the leading order Polyakov-loop effective action terms for the pure Yang-Mills sector. We have found double-peak structure in the Polyakov loop increasing rate $d \ell_{p} / d T$ and the susceptibility as a function of $T$ for small quark masses and large $\mu$. We have shown that the first and second peaks originate from the chiral and $Z_{N_{c}}$ symmetries. As future perspectives, we should evaluate higher order corrections of the Polyakov-loop effective action [17], and higher order terms of the strong coupling and $1 / d$ expansions. We expect that the current work gives a useful guide for the Monomer-Dimer-Polymer formulations [4, 7]. 


\section{Acknowledgements}

The authors thank to Maria Paola Lombardo, Lars Zeidlewicz, Philippe de Forcrand, Michael Fromm, Kim Splittorff and Zoltan Fodor for useful comments. This work was supported in part by Grants-in-Aid for Scientific Research from JSPS (No. 22-3314), the Yukawa International Program for Quark-hadron Sciences (YIPQS), and by Grants-in-Aid for the global COE program "The Next Generation of Physics, Spun from Universality and Emergence" from MEXT.

\section{References}

[1] For recent results and reviews, see, S. Borsanyi, Z. Fodor, C. Hoelbling, S. D. Katz, S. Krieg, C. Ratti and K. K. Szabo [Wuppertal-Budapest Collaboration], JHEP 1009 (2010) 073.

[2] The review of the pioneering works for the strong-coupling expansion (namely at $T=0$ ) is found in the text book, I. Montvay and G. Münster, "Quantum Fields on a Lattice,"

Cambridge University Press, 1994.

[3] P. H. Damgaard, N. Kawamoto and K. Shigemoto, Nucl. Phys. B 264 (1986), 1; P. H. Damgaard, D. Hochberg and N. Kawamoto, Phys. Lett. B 158, (1985) 239; G. Fäldt and B. Petersson, Nucl. Phys. B 265, (1986) 197; N. Bilic, F. Karsch and K. Redlich, Phys. Rev. D 45, (1992) 3228.

[4] F. Karsch and K. H. Mutter, Nucl. Phys. B 313, (1989), 541.

[5] Y. Nishida, K. Fukushima and T. Hatsuda, Phys. Rept. 398 (2004), 281; K. Fukushima, Prog. Theor. Phys. Suppl. 153 (2004), 204; Y. Nishida, Phys. Rev. D 69 (2004), 094501; V. Azcoiti, G. Di Carlo, A. Galante and V. Laliena, J. High Energy Phys. 09 (2003), 014.

[6] N. Kawamoto, K. Miura, A. Ohnishi and T. Ohnuma, Phys. Rev. D 75 (2007), 014502.

[7] P. de Forcrand and M. Fromm, Phys. Rev. Lett. 104 (2010) 112005.

[8] E. M. Ilgenfritz and J. Kripfganz, Z. Phys. C 29, (1985) 79; A. Gocksch and M. Ogilvie, Phys. Rev. D 31, (1985) 877; K. Fukushima, Phys. Rev. D 68, (2003) 045004.

[9] K. Fukushima, Phys. Lett. B 591 (2004), 277.

[10] K. Miura, T. Z. Nakano, A. Ohnishi and N. Kawamoto, Phys. Rev. D 80 (2009) 074034; K. Miura, T. Z Nakano and A. Ohnishi, Prog. Theor. Phys. 122 (2009), 1045.

[11] T. Z. Nakano, K. Miura and A. Ohnishi, Prog. Theor. Phys. 123 (2010) 825.

[12] T. Z. Nakano, K. Miura and A. Ohnishi, Phys. Rev. D 83 (2011) 016014; T. Z. Nakano, K. Miura and A. Ohnishi, PoS LATTICE2010 (2010) 205.

[13] K. Miura, T. Z. Nakano, A. Ohnishi and N. Kawamoto, PoS LATTICE2010 (2010) 202; K. Miura, T. Z. Nakano, A. Ohnishi and N. Kawamoto, arXiv:1106.1219 [hep-lat].

[14] T. Kahara and K. Tuominen, Phys. Rev. D 82 (2010) 114026; Phys. Rev. D 78 (2008) 034015; Phys. Rev. D 80 (2009) 114022.

[15] K. Fukushima, Phys. Lett. B 695, 387 (2011).

[16] T. K. Herbst, J. M. Pawlowski and B. J. Schaefer, Phys. Lett. B 696 (2011) 58.

[17] J. Langelage and O. Philipsen, JHEP 1004 (2010) 055; JHEP 1001 (2010) 089; J. Langelage, G. Munster and O. Philipsen, JHEP 0807 (2008) 036. 